\title{
ISPRS TECHNICAL COMMISSION IV MIDTERM SYMPOSIUM 2018 PREFACE
}

\author{
S. Zlatanova ${ }^{1 *}$, S. Dragicevic ${ }^{2}$, G. Sithole ${ }^{3}$ \\ ${ }^{1}$ President of TC IV, University of New South Wales, Sidney, Australia - s.zlatanova@unsw.edu.au \\ 2 Vice President of TC IV, Simon Fraser University, Burnaby, Canada - suzanad@sfu.ca \\ ${ }^{3}$ Secretary, University of Cape Town, Cape Town, South Africa - george.sithole@uct.ac.za
}

\section{Commission IV}

The world's population is predicted to reach 7.5 billion in 2018, and by 2050 it is predicted to be 9.7 billion. Managing the social and economic changes brought on by this increasing population will require new ideas and spatial information decision making tools. The pressures placed on the usage of spaces that people live and work in will necessitate decisions that are based on 3D and higher spatial dimensions. The Commission is dedicated to advance the research in spatial information sciences in modelling, structuring, management, analysis, visualization and simulation of data with focus on the third spatial dimension and taking into consideration dynamic changes. A special attention is given to linking information about real-world physical phenomena with societal, organizational and legal information. The intentions are to contribute to advancements to visualization (web-based, VR and AR) simulation and city analytics. The work largely carried out in cooperation with international organizations such as FIG, UDMS, 3DGeoinfo, ICA, OGC, ISO and Web3D.

The Commission consists of 10 scientific areas of research, which are coordinated by 10 working groups (WG) as follows - WG1: Strengthen the work on multidimensional spatial model and representations towards seam-less data fusion; WG2: Advance the semantic modelling, development and linking of ontologies; WG3: Intensify research into data interpretation, quality and uncertainty modelling; WG4: Strengthen the research on crowdsourced data and public participation, towards community-driven and participatory applications, collaborative mapping and use/usability of maps; WG5: Stringent the research on seamless indoor/outdoor location-based services, navigation and tracking, and analysis of human movement; WG6: Advance interoperable Internet of Things, Sensor web, SDI and linked data; WG7: Advance the research on spatial data types, indexing methods and analysis to further contribute to development of spatial DBMS for management and analysis of multi-dimensional data; WG8: Encourage the use of functional programming and streaming algorithms in development of demos and applications as well as parallel and distributed processing paradigms; WG9: Advance visual analytics, online multi-dimensional visualization on mobile and desktop devices, considering human-centered applications, privacy and security issues; WG10: Advance knowledge on use of spatial information (BIM/GIS) for urban modelling; ICWG IV/III: Global Mapping: Updating, Verification and Interoperability with the mission to promote the development of advanced methodologies and applications related to the update, verification and interoperability of geospatial databases. All these themes were presented in this year ISPRS TC IV Midterm Symposium 2018

The symposium was held in Delft, Netherlands, October 1-5, together with FIG - the 6th International Workshop on 3D Cadastres, UDMS - the 3rd International Conference on Smart Data and Smart Cities and the13th 3D GeoInfo Conference, all under the umbrella of GeoDelft 2018 conferences. These events have provided a synergy of the research communities from four related international organizations. Particularly, the aim of the ISPRS TC IV midterm symposiums was to bring together academics and professionals from industry working in the field of Spatial Information Sciences (SIS) to exchange the cutting-edge research and technological developments in this field. Moreover, the focuses outlined by the commission plan is to increase ISPRS TC IV exposure to diverse SIS research communities and to exchange the knowledge gained within its ten working groups and five intergroups. Five speakers Dorine Burmanje, Rudzhi Chen, Rod Thompson, Claus Nagel and Antonio Jara provided inspiring talks to enrich and share their experiences with the attendees. For all these reasons, the symposium was named 3D Spatial Information Science - The Engine of Change.

The symposium provided presenters and attendees with an engaging forum to communicate research findings, disseminate advancements in new and emerging theories, and demonstrate the applications and technologies of the spatial information sciences. The research presented at the event was captured through papers and abstracts published in the traditional proceedings of the 2018 ISPRS TC IV Midterm Symposium that includes the collection of ISPRS Annals and ISPRS Archive. The papers and abstracts were selected for inclusion through a rigorous peer-review process. The ISPRS Annals contain 36 papers and the ISPRS Archives consist of 108 papers. The diversity of the research topics presented in the published papers clearly indicate the wide range of topics within the field of Spatial Information Science. A rigorous peer-review process by the ISPRS TC IV conference Scientific Committee members ensured quality, diversity and innovation. The program consisted of 130 oral presentations and 14 posters. One of the sessions was jointly organised by OGC, SDSC and ISPRS and discussed recent interoperability developments. Three workshops were organized as follows: Theme session on Virtual \& Augmented Reality: Technology, Design \& Human Factors, Capacity Building for High-Resolution Land Cover Inter-comparison and Validation and OpenStreetMap mapathon. John Shi and Gerard Heuvelink, invited speakers at WG 3, discussed issue related to uncertainty in spatial modelling.

We would like to acknowledge the generous sponsorship support provided by Delft University of Technology, University of South Wales, Australia, University of Twente, Netherlands, University of Applied Sciences Stuttgart, FIG, UDMS, OGC and Geo-

\footnotetext{
* Corresponding author
} 
Information Netherlands. We are thankful to the members of the GeoDelft 2018 Organizing Committee, ISPRS TC IV Scientific and Organizing Committees, sponsors, exhibitors, students, volunteers and all those who have through their dedicated work contributed to a successful organization of the symposium.

\section{Conference Committees}

ISPRS TCIV Organizing Committee: Hussein M. Abdulmuttalib Dubai Municipality, Deira, Dubai UAE, Giorgio Agugiaro Austrian Institute of Technology, Vienna, Austria, Thomas Blaschke, University of Salzburg, Salzburg, Austria, Pawel Boguslawski Wroclaw University of Science and Technology, Wroclaw, Poland, Martin Breunig Karlsruhe Institute of Technology, Karlsruhe, Germany, Maria Antonia Brovelli Politecnico di Milano, Milan, Italy, Sidonie Christophe IGN-France, Paris, France, Arzu Coltekin University of Zurich, Zurich, Switzerland, Mahmoud R. Delavar University of Tehran, Tehran, Iran, Mulhim Al Doori American University, Dubai, UAE, Suzana Dragicevic Simon Fraser University, Burnaby, Canada, Eric Guilbert Université Laval, Québec, Canada, James Haworth University College London, London, United Kingdom, Guoman Huang Chinese Academy of Surveying and Mapping, Beijing, China, Umit Isikdag Mimar Sinan Fine Arts University Istanbul, Istanbul, Turkey, Mikhail Kanevski University of Lausanne, Lausanne, Switzerland, Zhizhong Kang China University of Geosciences, Beijing, China, Kourosh Khoshelham University of Melbourne, Melbourne, Australia, Mila Koeva University of Twente, Enschede, The Netherlands, Margarita Kokla National Technical University of Athens, Athens, Greece, Klaus Komp EFTAS Remote Sensing, Transfer of Technology, Muenster, Germany, Bart De Lathouwer Open Geospatial Consortium, Kontich, Belgium, Heather Leason OSM, Qatar, Yaolin Liu Wuhan University, Wuhan, China, Marguerite Madden University of Georgia, Athens, Georgia, USA, Mir Abolfazl Mostafavi Université Laval, Quebec, Canada, Gerhard Navratil Technical University Wien, Vienna, Austria, Dev Raj Paudyal University of Southern Queensland, Toowoomba, Australia, George Percivall Open Geospatial Consortium, Maryland, USA, Michael Peter University of Twente, Enschede, The Netherlands, Chris Pettit University of New South Wales, Sydney, Australia, George Sithole University of Cape Town, Cape Town, South Africa, Hao Wu National Geomatics Center of China, Beijing, China, Sisi Zlatanova University of New South Wales, Sydney, Australia

ISPRS TCIV Scientific Committee: Antonio Adan Universidad de Castilla La Mancha, Ciudad Real, Spain, Aida Afrooz The University of New South Wales, Sydney, Australia, Siddique Baig COMSATS Institute of Information Technology, Islamabad, Pakistan, Andrea Ballatore University of London, London, UK, Temenoujka Bandrova University of Architecture, Civil Engineering and Geodesy, Sofia, Bulgaria, Árpád Barsi Budapest University of Technology and Economics, Budapest, Hungary, Susanne Becker University of Stuttgart, Stuttgart, Germany, Mariana Belgiu University of Twente, Enschede, The Netherlands, Michela Bertolotto University College Dublin, Dublin, Ireland, Filip Biljecki National University of Singapore, Singapore, Jan Blachowski Wrocław University of Science and Technology, Wroclaw, Poland, Jack Barton University of New South Wales, Sydney, Australia, Thomas Blaschke University of Salzburg, Salzburg, Austria, Lars Bodum Aalborg University, Aalborg, Denmark, Bénédicte Bucher IGN, France, Christos Chalkias Harokopio University of Athens, Greece, Jiangping Chen Wuhan University, Wuhan, China, Sidonie Christophe IGN-France, Paris, France, Christophe Claramunt Naval Academy Research Institute, France, Eliseo Clementini University of L'Aquila, L'Aquila, Italy, Serena Coetzee University of Pretoria, Pretoria, South Africa, Arzu Coltekin University of Zurich, Zurich, Switzerland, Sophie Crommelinck University of Twente, Enschede, The Netherlands, Youness Dehbi Bonn University, Bonn, Germany, Lucía Díaz Vilariño University of Vigo, Vigo, Spain, Andrew Dempster The University of New South Wales, Sydney, Australia, Abdoulaye Diakité University of New South Wales, Sydney, Australia, Shihong Du Peking University, Beijing, China, Edward Duncan The University of Mines \& Technology, Tarkwa, Ghana, Claire Ellul University College London, London, UK, Cidália C. Fonte University of Coimbra, Coimbra, Portugal, Caroline M. Gevaert University of Twente, Enschede, The Netherlands, Tarun Ghawana Integrated Spatial Analytics Consultants, Ben Gorte The University of New South Wales, Sydney, Australia, George Grekousis Sun Yat-Sen University, Guangdong, China, Amy Griffin Royal Melbourne Institute of Technology, Melbourne, Australia, Ihab Hijazi An-Najah National University, Nablus, Palestine, Charlotte Hoarau IGN France, France, Guoman Huang Chinese Academy of Surveying and Mapping, Beijing, China, Frédéric Hubert Université Laval, Québec, Canada, Umit Isikdag Mimar Sinan Fine Arts University, Istanbul, Turkey, Halldor Janetzko University of Zurich, Zürich, Switzerland, Peng Jiang University of New South Wales, Sydney, Australia, Martin Kada Technical University of Berlin, Berlin, Germany, Mohsen Kalantari The University of Melbourne, Melbourne, Australia, Ismail Rakip Karas Karabük University, Karabük, Turkey, Marinos Kavouras National Technical University of Athens, Athens, Greece, Kourosh Khoshelham University of Melbourne, Parkville, Australia, Thomas Kolbe Technical University of Munich, Munich, Germany, Klaus Komp EFTAS Remote Sensing, Transfer of Technology, Muenster, Germany, Milan Konecný Masaryk university, Brno, Czech Republic, Tatjana Kutzner Technical University of Munich, Munich, Germany, Paul Vincent Kuper Karlsruhe Institute of Technology, Karlsruhe, Germany, Simone Z Leao The University of New South Wales, Sydney, Australia, Hugo Ledoux Delft University of Technology, Delft, The Netherlands, Binghao Li The University of New South Wales, Sydney, Australia, Fangyu Li China University of Petroleum in Beijing, Beijing, China, Ki-Joune Li Pusan National University, South Korea, Yaolin Liu Wuhan University, Wuhan, China, Ali Mansourian Lund University, Lund, Sweden, Martijn Meijers Delft University of Technology, Delft, The Netherlands, Marco Minghini Politecnico di Milano, Milan, Italy, Mir Abolfazl Mostafavi Université Laval, Quebec, Canada, Masafumi Nakagawa Shibaura Institute of Technology, Tokyo, Japan, Francesca Noardo Politecnico di Torinom, Torino, Italy, Ken Arroyo Ohori Delft University of Technology, Delft, The Netherlands, Frank Ostermann Faculty of Geo-Information Science and Earth Observation (ITC), Enschede, The Netherlands, George Percivall Open Geospatial Consortium, Maryland, USA, Claudio Persello University of Twente, Enschede, The Netherlands, Alan Peters The University of New South Wales, Sydney, Australia, Stefan Peters University of South Australia, Adelaide, Australia, Alenka Poplin Iowa State University, Ames, USA, Alias Abdul Rahman University of Technology Malaysia, Kuala Lumpur, Malaysia, Ana-Maria Olteanu-Raimond French Mapping Agency, France, Victoria Rautenbach University of Pretoria, Pretoria, South Africa, Anthony Robinson The Pennsylvania State University, State College, 
United, Éric Saux École Navale, France, Linda See International Institute for Applied Systems Analysis, Laxenburg, Austria, Jianga Shang China University of Geosciences, China, Antonia Spanò Politecnico di Torino, Torino, Italy, Emmanuel Stefanakis University of New Brunswick, Fredericton, Canada, Eleni Tomai National Technical University of Athens, Athens, Greece, Guillaume Touya IGN, Paris, France, Wei Tu Shenzhen University, Shenzhen, China, Giuseppina Vacca University of Cagliari, Cagliari, Italy, Corné van Elzakker University of Twente, Enschede, The Netherlands, Bert Veenendaal Curtin University, Perth, Australia, Edward Verbree Delft University of Technology, Delft, The Netherlands, Maria Vasardani The University of Melbourne, Melbourne, Australia, Mingshu Wang University of Georgia, Athens, Georgia, USA, Shuangfeng Wei Beijing University of Civil Engineering and Architecture, Beijing, China, Stephan Winter The University of Melbourne, Melbourne, Australia, Sarah Wise University College London, London, United Kingdom, Shen Ying Wuhan University, Wuhan, China, Ling Zhang Nanjing Normal University, Nanjing, China, Rui Zhu Singapore-MIT Alliance for Research and Technology, Singapore, Qing Zhu, Southwest Jiaotong University, China, Xianfeng Zhang Peking University, Beijing, China, John Shi Wen-Zhong The Hong Kong Polytechnic University, Hong Kong. 